\title{
Association between angiotensin II receptor gene polymorphism and serum angiotensin converting enzyme (SACE) activity in patients with sarcoidosis
}

Yuji Takemoto, Mitsunori Sakatani, Seijyu Takami, Teruo Tachibana, Jitsuo Higaki, Toshio Ogihara, Tetsuro Miki, Tomohiro Katsuya, Tessei Tsuchiyama, Akira Yoshida, Hisahiro Yu, Yoshiro Tanio, Einosuke Ueda

Department of
Medicine, National
Kinki Central
Hospital, Nagasonecho
1180, Sakai City, Osaka
591, Japan
Y Takemoto
M Sakatani
T Tsuchiyama
H Yu
A Yoshida
E Ueda

Department of Geriatric Medicine, Osaka University Medical School, 2-2 Yamadaoka, Suita City, Osaka, 565, Japan

S Takami

J Higaki

T Miki

T Katsuya

T Ogihara

Department of Medicine, Osaka Prefectural Hospital, Manndai Higashi 3-1-56, Sumiyosu-ku, Osaka City, 558 Japan

$\mathrm{T}$ Tachibana

$\mathrm{T}$ Tanio

Correspondence to: Dr Y Takemoto.

Received 15 September 1997 Returned to author 24 November 1997

Revised manuscript received 3 February 1998

Accepted for publication

9 February 1998

\begin{abstract}
Background-Serum angiotensin converting enzyme (SACE) is considered to reflect disease activity in sarcoidosis. SACE activity is increased in many patients with active sarcoid lesions. The mechanism for the increased SACE activity in this disease has not been clarified. ACE insertion/deletion (I/D) gene polymorphism has been reported to have an association with SACE levels in sarcoidosis, but no evidence of an association between angiotensin II receptor gene polymorphism and SACE in this disease has been found. A study of the association of angiotensin II receptor gene polymorphisms with sarcoidosis was therefore undertaken.

Methods-ACE (I/D), angiotensin II type 1 receptor (AGTR1), and angiotensin II type 2 receptor (AGTR2 ) gene polymorphisms were investigated by polymerase chain reaction (PCR) and SACE levels were measured in three groups of patients: those with sarcoidosis or tuberculosis and normal controls.

Results-There was no difference in allele frequency of AGTR1 and AGTR2 polymorphism among the three groups. Neither AGTR1 nor AGTR2 polymorphisms were associated with sarcoidosis. SACE activity was higher in patients with sarcoidosis with the AGTR1 A/C genotype than in others. However, this tendency was not detected in patients with tuberculosis. Conclusions-The AGTR1 allele C is associated with high activity of SACE in patients with sarcoidosis. It is another predisposing factor for high levels of SACE in patients with sarcoidosis and is considered to be an independent factor from the ACE D allele for high levels of SACE in sarcoidosis. This fact could be one of the explanations for the increased SACE activity in sarcoidosis.

(Thorax 1998;53:459-462)
\end{abstract}

Keywords: angiotensin II receptor gene; polymorphism; angiotensin converting enzyme

Sarcoidosis is a chronic multisystem disorder of unknown aetiology characterised in affected organs by accumulation of $\mathrm{T}$ lymphocytes, mononuclear phagocytes, and non-caseating epithelioid granulomas. High serum angiotensin converting enzyme (SACE) activity has been reported specifically in patients with sarcoidosis ${ }^{1}$ and is considered to reflect disease activity. ${ }^{2}$ High SACE activity in patients with sarcoidosis is associated with a poorer prognosis than those with lower activity. ${ }^{3}$ However, the precise mechanism of increased SACE activity in this disease has not yet been clarified.

Genetic polymorphism studies in many diseases have recently revealed new aspects of clinical importance. ACE I/D (insertion and deletion) polymorphism has also been studied and the ACE D allele is reported to be a predisposing factor for high SACE levels in patients with sarcoidosis. ${ }^{4}$ ACE is an enzyme which converts angiotensin I to angiotensin II and the new formed angiotensin II then acts on angiotensin receptors. Two types of angiotensin receptors have been cloned in humans (type 1 (AGTR1) and type 2 (AGTR2)), and their polymorphism and sequences have been identified. $^{5-7}$ AGTR1 A/C $\mathrm{C}^{1166}$ polymorphism represents the transition of $\mathrm{A}^{1166}$ to $\mathrm{C}$, which is an untranslated region and may be nonfunctional. The clinical significance of this polymorphism has not so far been studied but it might have some relation to SACE levels in this disease. In this study we have investigated whether some displacement of these receptor polymorphisms occurs specifically in patients with sarcoidosis.

\section{Methods}

SUBJECTS

One hundred patients with sarcoidosis, 50 patients with pulmonary tuberculosis, and 247 normal control subjects were studied. All patients were Japanese and the mean (SD) ages of those with sarcoidosis and tuberculosis were 49.4 (12.7) and 51.6 (15.2), respectively. Patients were followed up in National Kinki Central Hospital and Osaka Prefectural Hospital from July 1996 to July 1997. Two hundred and forty seven normal subjects were analysed at the Department of Geriatric Medicine, Osaka University, 96 of whom were used as controls in the study of an association between SACE levels and genetic polymorphisms. The diagnosis of sarcoidosis was based on clinical findings and histological findings of noncaseating granulomas, and a diagnosis of pulmonary tuberculosis was based on the 
Table 1 Allele frequencies and SACE activity of subgroups of ACE, AGTR1 and AGTR2 polymorphisms in subjects with sarcoidosis, subjects with tuberculosis and normal subjects

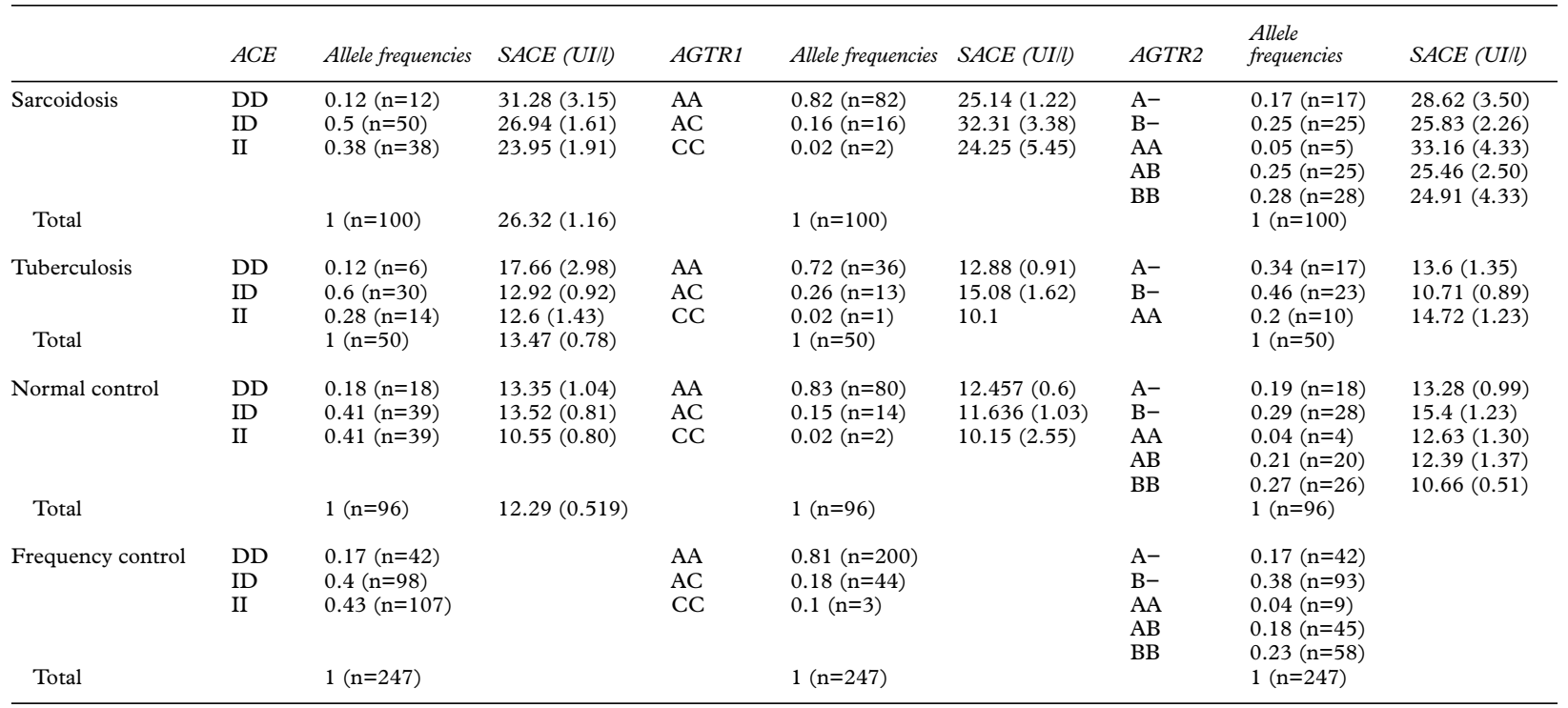

$\mathrm{n}=$ numbers of subjects.

presence of Mycobacterium tuberculosis in the sputum or histological findings of caseating granulomas. Patients who were receiving ACE inhibitors or antihypertensive drugs were excluded from the study.

ACE ASSAY

ACE activity was assayed photometrically using P-hydroxybenzoyl-glycyl-L-histidyl-Lleucine as the substrate according to the method of Kasahara et al (ACE colour, Fujirebio, Inc, Tokyo, Japan). ${ }^{8}$

ANALYSIS OF ACE, AGTR1 AND AGTR2 GENE POLYMORPHISM

Total DNA was purified from leucocytes taken from each subject using the phenol/ chloroform method. Primers for ACE, AGTR1, and AGTR2 gene amplification were synthesised by Sawady Co (Japan) and the following primer sequences were used: ACE, sense oligo 5'-CTGGAGACCACTCCCATC CTTTCT-3', antisense oligo 5'-GATGTGGC CATCACATTCGTCAGA-3'; ATGR1, sense oligo 5'-ATAATGTAAGCTCATCCACC-3', antisense oligo 5'-GAGATTGCATTTCTG TCAGT-3'; ATGR2, sense 5'-GGATTCAGA TTCTCTTTGAA-3' and antisense oligo 5'GCATAGGAGTATGATTTAATC-3'. PCR was performed under standard conditions. The reaction mixture for ACE gene was amplified in the following steps: denaturation at $94^{\circ} \mathrm{C}$ for 60 seconds, annealing at $55^{\circ} \mathrm{C}$ for 30 seconds and extension at $72^{\circ} \mathrm{C}$ for 90 seconds which was repeated 35 times by thermal cycler (Perkin Elmer Cetus, USA). For AGTR1 each cycle of the reaction consisted of denaturation for 30 seconds at $94^{\circ} \mathrm{C}$, annealing at $53^{\circ} \mathrm{C}$ for 45 seconds, and extension at $72^{\circ} \mathrm{C}$ for 45 seconds which was repeated 40 times, and for AGTR2 each cycle of the reaction consisted of denaturation at $94^{\circ} \mathrm{C}$ for 30 seconds, annealing at $53^{\circ} \mathrm{C}$ for 45 seconds, and extension at $72^{\circ} \mathrm{C}$ for 45 seconds which was repeated 40 times. Ten $\mu \mathrm{l}$ of ACE gene reaction mixture was added to $2 \%$ Nusieve agarose gel (FMC Bioproducts, USA) without enzyme digestion. Electrophoresis was performed using $1 \times \mathrm{TAE}$ solution. Ten $\mu$ l of AGTR1 and AGTR2 reaction mixture were digested with 10 units of Dde I and of Alu I each for an hour at $37^{\circ} \mathrm{C}$. Each solution was added to $2 \%$ Nusieve agarose gel and electrophoresis was performed. DNA was stained with ethidium bromide and banding patterns were determined and then photographed under UV radiation.

A 350 base pair (bp) fragment was amplified with AGTR1 primers and divided into two fragments (211 bp and $139 \mathrm{bp}$ ) by digesting it with Dde I restriction enzyme. A 350 bp fragment was named allele $A$ and a $211 \mathrm{bp}$ fragment was named allele C. A $312 \mathrm{bp}$ fragment was amplified and digested to $215 \mathrm{bp}$ and $107 \mathrm{bp}$ fragments by Alu I. The $312 \mathrm{bp}$ and $215 \mathrm{bp}$ fragments were named alleles $\mathrm{A}$ and $\mathrm{B}$, respectively.

ACE (I/D), AGTR1, and AGTR2 gene polymorphism in the three groups (patients with sarcoidosis, tuberculosis and normal controls) was assessed by the $\chi^{2}$ and ANOVA (analysis of variance) tests. Relationships of SACE to polymorphisms (ACE (I/D), AGTR1 and AGTR2) were analysed by ANOVA of a StatView J-4.5 application program (Abacus, USA). A post hoc (Fisher's PLSD test) and two sample $t$ tests were applied for further analysis of significant results from the ANOVA test.

\section{Results}

The frequency of ACE polymorphism in patients with sarcoidosis was not significantly different from that in control subjects and those with tuberculosis (table 1), nor was there a significant difference in the frequency of AGTR1 polymorphism between the three groups (table 1). A difference in allele frequency appeared in AGTR2 polymorphism between patients with tuberculosis and control subjects $\left(\chi^{2}=45.02, \mathrm{p}<0.001\right)$ but no 
significant difference was seen between patients with sarcoidosis and controls $\left(\chi^{2}=5.88\right.$, $\mathrm{p}=0.208)$. When only men were considered the significance of the difference was lost $\left(\chi^{2}=\right.$ $0.101, p=0.751)$. No significant association was detected between sarcoidosis and each gene polymorphism (ACE I/D (odds $=1.07$ ), AGTR1 (odds = 1.713), and AGTR2 (odds = 1.511)).

Mean SACE activity in patients with sarcoidosis was 26.32 (1.16) IU/1 which was significantly higher than in those with tuberculosis (13.47 (0.78) IU/1) and in normal controls (12.29 (0.519) IU/1); p<0.001 (ANOVA). SACE activity in the DD genotype was high compared with the ID and II genotypes but this difference was not significant $(\mathrm{p}=0.1037)$. These tendencies were also seen in patients with tuberculosis $(\mathrm{p}=0.1321)$ and were significant in normal subjects $(p=0.026)$. These results resemble those of Furuya et $a l^{4}$ concerning an association between SACE activity and ACE polymorphism. Subjects with the AGTR1 C allele who had sarcoidosis had significantly higher SACE levels $(p=0.043$ AA vs $A C+C C)$ than those with tuberculosis $(p=$ 0.299 AA vs AC + CC) and control subjects ( $p$ $=0.477 \mathrm{AA}$ vs AC + CC ) by two sample $t$ tests but the ACE D allele showed a tendency to be associated with SACE levels in all three groups. To compare SACE levels in the presence and absence of the AGTR1 allele C two sample $t$ tests and Fisher's PLSD test were performed. SACE activity in patients with sarcoidosis having the AGTR1 AC genotype ( $p=0.025$ AA vs $\mathrm{AC}$ ) showed a significant difference compared with other genotypes of ATGR1. No association between AGTR2 genotypes and SACE levels was found in the three groups of patients (sarcoidosis $(\mathrm{p}=0.128)$; tuberculosis $(\mathrm{p}=$ 0.233 ); and normal controls $(\mathrm{p}=0.1488)$ ) by ANOVA. Analysis of combined groups of ACE and AGTR1 polymorphism revealed that both ACE and AGTR1 polymorphism had no synergistic effect on the increase in SACE activity in patients with sarcoidosis. Subjects with the ACE DD genotype had higher SACE activity (34.73 (3.94) IU/1) than the other ACE genotypes (ID, 26.26 (1.70) IU/1; II, 21.51 (1.72) IU/1) in the subgroup with the AGTR1 AA genotype. However, these tendencies were not seen in the subgroup with the AGTR1 AC genotype. SACE levels in patients with the ACE DD genotype (25.90 (3.55) IU/1) were lower than the other ACE genotypes (ID, 32.92 (5.08) IU/1, II, 34.18 (6.87) IU/l) in the subgroups with the AGTR1 AC genotype (non-significant). ACE polymorphism showed an inverse effect on SACE levels in the subgroups of AGTR1 polymorphism.

\section{Discussion}

The ACE D allele is reported to be a predisposing factor to high SACE levels both in normal subjects and in patients with sarcoidosis. ${ }^{4}$ In our study of Japanese patients with sarcoidosis, tuberculosis, and normal controls the ACE D allele was also associated with higher SACE levels than the ACE I allele. SACE levels differ among races which may be due to the heterogeneity of ACE I/D polymorphism. Black patients with sarcoidosis have higher SACE activity than white subjects and Japanese. ${ }^{9}$ Such an association has not explained the mechanism of high SACE activity in patients with sarcoidosis, but ACE I/D polymorphism may be one of the genetic factors responsible for this. From a study of familial clustering, high prevalence and clinical severity of sarcoidosis in African Americans Rybiki et al concluded that the heterogeneity of the risk of familial sarcoidosis might have several aetiologies. ${ }^{10}$

The importance of AGTR1 polymorphism has been reported in addition to ACE polymorphism in cardiovascular disease, hypertension, and arteriosclerosis. ${ }^{11}{ }^{13}{ }^{14}$ Here we have noted a significant increase in SACE activity in patients with AGTR1 AC genotype $(\mathrm{p}<0.05)$ compared with AA genotype and CC genotype (table 1). The frequency of AGTR1 CC genotype (2\%) was too infrequent for statistical analysis, as Tiret has reported previously. ${ }^{11}$ AGTR1 A/C $\mathrm{C}^{1166}$ polymorphism in patients with sarcoidosis shows high SACE activity, in contrast to patients with tuberculosis and normal controls. We conclude that the presence of the AGTR1 C allele (AC or CC genotypes) has a specific association with SACE levels in patients with sarcoidosis $(p<0.05)$. Changes of amino acid at polymorphic sites in ATGR1 are not associated with cardiovascular disease and no mutation in the coding region of AGTR1 was identified in a group of 50 hypertensive patients. ${ }^{12}$ SACE activity does not appear to predispose to hypertension in patients with sarcoidosis. This suggests that the angiotensin II signal may not be conveyed, so the association of the AGTR1 $\mathrm{C}$ allele with sarcoidosis may involve functional mutations in the coding sequences of AGTR1.

Tiret et al reported that the AGTR1 C allele substantially increases the effect of ACE DD genotype on the risk of myocardial infarction in a low risk group. ${ }^{11}$ Bonnardeaux et al found that the ACE I allele is weakly associated with aortic elasticity in hypertension and the AGTR1 C allele is a strong determinant of this. ${ }^{13}$ The ACE D allele shows less SACE activity than the ACE I allele in subjects with sarcoidosis who have the AGTR1 C allele and the opposite is true in a subgroup with the AGTR1 A allele. Both ACE and AGTR1 polymorphisms which favour an increase in SACE activity in patients with sarcoidosis may have an inverse effect rather than a synergistic one. Analysis of AGTR2 polymorphism does not disclose any association in patients with sarcoidosis. In patients with tuberculosis the significant difference in allele frequency in AGTR2 is dependent on sex because AGTR2 is located on the X chromosome. The functions of AGTR1 are threefold: breakdown of phosphoinositide by stimulation of phospholipase $\mathrm{C}$ resulting in the release of intracellular $\mathrm{Ca}^{2+}$, activation of voltage dependent $\mathrm{Ca}^{2+}$ channels, and inhibition of adenylate cyclase activity. ${ }^{14}$ The function of AGTR2 is known to be different from AGTR1 but it has not been clearly identified. AGTR2 polymorphism does not affect SACE activity in patients with sarcoidosis. 
In conclusion, the results of our study support the findings that AGTR1 polymorphism manifests an additional predisposition to high SACE activity in patients with sarcoidosis. The AGTR1 C allele is associated with SACE in patients with sarcoidosis and affects SACE levels independently of the ACE D allele. Further studies are warranted to clarify the possibility of a link between mutations on AGTR1 and high SACE activity in patients with sarcoidosis.

1 Lieberman J. Elevation of serum angiotensin converting enzyme (ACE) level in sarcoidosis. Am F Med 1975;59:36572 .

2 Famburg BL, Friedland J, Lyan HA, et al. Elevated serum angiotensin converting enzyme in granulomatous lymph nodes and serum in sarcoidosis. Am Rev Respir Dis 1976;114:525-8.

3 Ueda E, Kawabata T, Tachibana T, et al. Serum angiotensin converting enzyme activity as an indicator of prognosis in sarcoidosis. Am Rev Respir Dis 1980;121:667-71

4 Furuya K, Yamaguchi E, Itoh A, et al. Deletion polymorphism in the angiotensin converting enzyme (ACE) gene as a genetic risk factor for sarcoidosis. Thorax 1996;51:77780 .

5 Katsuya T, Koike G, Thomas WY, et al. Association of angiotensinogen gene T235 variant with increased risk of tensinogen gene T235 variant with increase
6 Katsuya T, Horiuchi M, Minami S, et al. Genomic organization and polymorphism of human angiotensin II type 2 receptor: no evidence for its gene mutation in two families of human premature ovarian failure syndrome. Mol Cell Endocrinol 1997;127:221-8.

7 Takayanagi R, Ohnaka K, Sakai Y, et al. Molecular cloning human type-1 angiotensin receptor. Biochem Biophys Res Commun 1992;183:910-6

8 Kasahara Y, Ashihara Y. Colorimetry of angiotensin-I converting enzyme activity in serum. Clin Chem 1981;27: 1922-25.

9 Lieberman J, Nosal A, Schlessner LA, et al. Serum angiotensin-converting enzyme for diagnosis and therapeutic elevation of sarcoidosis. Am Rev Respir Dis 1979;120: 329-35.

10 Rybicki BA, Harrington D, Major M, et al. Heterogeneity of familiar risk in sarcoidosis. Genet Epidemiol 1996;13:23-33.

11 Tiret L, Bonnardeaux A, Poirier O, et al. Synergistic effects of angiotensin converting enzyme and angiotensin-II type 1 receptor gene polymorphisms on risk of myocardial infarction. Lancet 1994;344:910-3.

12 Rolfs A, Rolfs IW, Zagrozek VR, et al. Genetic polymorphism of the angiotensin II type 1 (ATT) receptor gene. Eur Heart f 1994;15:108-12.

13 Bonnardeaux A, Davies E, Jeunematre X, et al. Angiotensin II type 1 receptor gene polymorphisms in human essential hypertension. Hypertension 1994;24:63-9.

14 Badenhop RF, Wang X Li, Wilcken DEL. Association between an angiotensinogen microsatellite marker in children and coronary events in their grandparents. Circulation 1996;93:2092-6.

15 Kakar SS. Angiotensin II type 1 receptors: molecular cloning and gene expression. Ketsuatu 1995;2:149-64. 